\title{
Inhibition of CD203c membrane up-regulation in human basophils by high dilutions of histamine: a controlled replication study
}

\author{
Salvatore Chirumbolo · Maurizio Brizzi • \\ Riccardo Ortolani · Antonio Vella • \\ Paolo Bellavite
}

Received: 14 November 2008/Revised: 3 April 2009/Accepted: 9 April 2009/Published online: 6 May 2009 (C) The Author(s) 2009. This article is published with open access at Springerlink.com

\begin{abstract}
Objective Previous research suggests that human basophil activation may be inhibited by histamine even at extremely low doses (high dilutions). However, uncertainties about the nature of the phenomenon and its reproducibility mean that further, rigorously controlled studies are necessary.

Methods Serial 1:100 (v:v) histamine dilutions (centesimal dilutions, C) and water controls were tested on human basophil responsiveness to anti-IgE antibodies, using flow cytometry. Each dilution step was followed by vertical mechanical shaking (also designed as succussion) at 20 strokes/s. Basophil-enriched buffy coats from healthy blood donors were incubated with $10^{-4} \mathrm{~mol} / \mathrm{l}$ histamine (2C) and with serially diluted preparations from $10^{-20} \mathrm{~mol} / \mathrm{l}(10 \mathrm{C})$ to $10^{-32} \mathrm{~mol} / \mathrm{l}(16 \mathrm{C})$, then incubated for 30 min with $1 \mu \mathrm{g} / \mathrm{ml}$ goat monoclonal anti-human $\operatorname{IgE}$ and basophils stained for immunophenotyping.

Results Membrane up-regulation of CD203c, which in these experimental conditions proved to be a more consistent activation marker than CD63, was significantly
\end{abstract}

Responsible Editor: A. Falus.

S. Chirumbolo $\cdot$ P. Bellavite $(\square)$

Department of Morphological and Biomedical Science,

University of Verona, Piazza L.A: Scuro, 10,

37134 Verona, Italy

e-mail: paolo.bellavite@univr.it

M. Brizzi

"P. Fortunati" Institute of Statistics, University of Bologna,

Bologna, Italy

R. Ortolani · A. Vella

Department of Pathology-Immunology Section,

University of Verona, Verona, Italy inhibited in samples treated with histamine at the dilutions of 2C $(P=0.001), 12 \mathrm{C}(P=0.047), 14 \mathrm{C}(P=0.003)$, 15C $(P=0.036)$ and $16 \mathrm{C}(P=0.009)$. Control water dilutions/succussions did not show any significant effect.

Conclusion Using a strictly standardized flow cytometry protocol and a new dilution/succussion procedure, we have shown that low and high dilutions of histamine inhibit CD203c up-regulation in anti-IgE stimulated basophils.

Keywords Basophil activation - Histamine ·

High dilutions · CD203c up-regulation · Ultra low doses

\section{Introduction}

In the past 20 years, many laboratory studies on cell [1] and animal [2] models have sought to test the pharmacological effects of dilutions which statistically contain very few or no molecules of active principle. The most widely investigated high-dilution effect has been the regulation of basophil leukocytes. A prominent multicentre study [3] found that human basophils underwent "degranulation" (detected as cell metachromasia) not only at the usual antiIgE antibody doses $\left(10^{-3} \mathrm{mg} / \mathrm{ml}\right)$, but also at extremely high dilutions of antibody ("sub-molecular" doses). This work was published in the journal "Nature" and had considerable resonance as a presumed demonstration of "water memory", but was heavily criticized on theoretical grounds and due to the difficulty of reproducing the results [4-6]. Although this particular model was not followed up by further replication studies, there has been extensive research on basophil response to highly diluted substances focused on the effects of histamine. Several authors report the inhibition of basophil "degranulation", evaluated by optical microscopy of cells stained with alcian or toluidine 
blue, using high dilutions of pure histamine at theoretical concentrations ranging from $10^{-10} \mathrm{~mol} / \mathrm{l}$ (5th centesimal dilution, 5C) to $10^{-38} \mathrm{~mol} / 1$ (19th centesimal dilution, 19C) [7-9]. The high-dilution effect of histamine has also been confirmed in a multicentre trial [10], although the authors report that not all the laboratories involved were able to observe the phenomenon, possibly due to variations in basophil sensitivity depending on the donors [11]. Controversies over the manual method used to assess basophil activation, along with its time-consuming and subjective nature, prompted adoption of a standardized automated flow cytometry approach [12], which was able to evaluate the effect of highly diluted compounds by quantitatively following membrane expression of the investigated cells. More recently, similar effects have been confirmed by two independent laboratories [13, 14] and by the group of Belon and Sainte-Laudy [15, 16], who also report that $\mathrm{CD} 203 \mathrm{c}$ is the most suitable marker for this type of effect [17]. However, the literature in this field is not always consistent, with some experiments showing that highly diluted histamine activates rather than inhibiting the same cells [18], while another group failed to replicate the reported inhibitory effects of high histamine dilutions [19]. These last-mentioned authors suggest that minor variables of the experimental set-up, such as the placement of samples in different rows on the same laboratory microplate, may produce significant differences in the results if not properly controlled (though it is worth noting that most flow cytometry experiments performed by the other authors were done using disposable tubes). Finally, a number of studies have used only a $10^{-32} \mathrm{~mol} / \mathrm{l}$ solution (16C) [16], or a pool of several dilutions [10], but dose-response curves are rarely reported [13].

Notwithstanding the prevailing evidence in favor of a true "high-dilution effect", the implausibility of such claims calls for serious investigation into the possibility of artifacts, arising from as-yet unidentified methodological factors, also because the research in this field has until now failed to account for the discrepancies observed between different laboratories. Even where many experiments of high methodological standard have demonstrated an effect of high dilutions, there has been no positive result stable enough to be reproduced by all investigators [20-24]. Therefore, given the profound pharmacological and biological implications of high-dilution effects, further independent replications, under rigorously controlled experimental conditions, are needed [25].

The aim of this study was to further investigate the effects of histamine on human basophils in vitro, using a new polychromatic flow cytometry protocol to quantify membrane expression of basophil activation markers [26]. Cell activation or inhibition were detected by the mean fluorescence intensity (MFI) of CD203c, a membrane ectoenzyme which represents the most sensitive IgE-specific basophil activation marker [26-28], and by the percentage of cells which up-regulated CD63, a molecule which is associated to the lysosomes and is involved in degranulation events [29]. Basophil activation results in a dose-dependent increase in CD203c MFI, while expression of CD63 is an all-or-nothing phenomenon which occurs at relatively higher doses of stimuli and exhibits bimodal distribution [26, 30], with one peak of highly positive cells (being the FITC fluorochrome highly fluorescent) and another one of fully negative cells. This is the reason why most researchers prefer to evaluate CD63 up-regulation not by the MFI, but by the percentage of cells that express the marker compared to the non-expressing resting state [11-16].

Here, we report the results of a series of experiments, in which particular care was taken with the following technical details: (a) to create favorable conditions for the putative inhibitory effects of histamine, cells were activated using a relatively low dose of anti-IgE, namely $1 \mu \mathrm{g} / \mathrm{ml}$ [26]; (b) both low (2C) and high (10C to 16C) dilutions of histamine were tested, thus in a range encompassing the Avogadro constant $\left(6.022 \times 10^{-23} \mathrm{~mol} / \mathrm{l}\right)$; (c) each dilution step involved a $100 \times$ decrease in concentration, and was followed by vigorous mechanical agitation ("succussion" according to homeopathic pharmacopoeia) in a shaking machine commonly used in pharmaceutical practice; (d) all the assays were performed in triplicate tubes in sterile conditions and using disposable plasticware to decrease the risk of cross-contamination; (e) ultrapure distilled water was used as a solvent, and a series of succussed dilutions of pure water were run to control for artifacts arising from the procedures or the materials employed; (f) in order to test the two main variables, namely CD203c MFI and percentage of CD63 positive (bright) cells, we used quantitative analyses and objective evaluations, a procedure that makes performing experiments in blinded fashion less necessary as it would be when performing the optical detection of basophil degranulation.

\section{Materials and methods}

Reagents and disposable ware

4-(2-Hydroxyethyl)piperazine-1-ethanesulonic acid (HEPES), sodium heparine $170 \mathrm{U} / \mathrm{mg}$, histamine dihydrochloride, and salts were purchased from Sigma-Aldrich, GmbH, Germany. Water used for stock solution and dilutions was purchased from Sigma-Aldrich as HPLC-grade water (Chromasolv plus) $(<0.001 \%$ non-volatile matter). Histamine dihydrochloride was prepared as a stock solution of $0.4 \mathrm{~mol} / \mathrm{l}$ in water. Anti-human goat monoclonal $\mathrm{IgE}$ was sourced from Caltag, USA. Anti-CD203c-PE (isotype 
IgG1, clone 97A6) was purchased from Beckman Coulter Immunotech, USA, anti-CD123-PECy5 (isotype IgG1, clone 9F5), anti-HLA-DR-PECy7 (isotype IgG2a, clone L243), anti-CD45-APCCy7 (isotype IgG1, clone 2D1) and anti-CD63-FITC (isotype IgG1, clone H5C6) were purchased from Becton Dickinson Pharmingen USA. All reagents were prepared using sterile disposable plastic tubes and pipette tips (BD Falcon, NJ, USA).

\section{Dilution and succussion procedures}

Dilutions were made up in $15 \mathrm{ml}$ sterile polystyrene conical tubes provided with caps; the serial dilutions were prepared with an actual histamine concentration four times greater than the intended final concentration, because the protocol subsequently called for a further fourfold dilution in the cell incubation mixture (see below). A $4 \times 10^{-2} \mathrm{~mol} / \mathrm{l}$ histamine solution (designated $1 \mathrm{C}$ ) was made by diluting $500 \mu \mathrm{l}$ of histamine stock in $4,500 \mu \mathrm{l}$ of ultrapure water. From that preparation, a series of incremental $1 / 100$ dilutions were prepared by diluting $50 \mu \mathrm{l}$ of the preceding solution in $4,950 \mu \mathrm{l}$ of ultrapure water and shaking by vigorous mechanical shaking $(7.5 \mathrm{~s}$ at 20 strokes/s, vertical amplitude $7 \pm 2 \mathrm{~mm}$ ) using an instrument from MGA Technologies, Lyon, France. The chosen working dilutions/succussions were $2 \mathrm{C}\left(10^{-4} \mathrm{~mol} / \mathrm{l}\right.$ histamine in the final sample assay) as a positive control, and seven ultra high dilutions from $10 \mathrm{C}\left(10^{-20} \mathrm{~mol} / \mathrm{l} \mathrm{his-}\right.$ tamine in the final sample assay) to $16 \mathrm{C}\left(10^{-32} \mathrm{~mol} / \mathrm{l}\right.$ histamine in the final sample assay). Experiments with high water dilutions were performed using control test samples made up by an identical procedure, with the only difference that the stock starting solution was pure water rather than histamine. The histamine and water control dilutions/succussions were prepared fresh each day just before the experiments, and stored at room temperature and protected from light until use.

\section{Cell preparation}

Basophil-enriched cell samples were prepared by pooling the leukocyte buffy coats drawn from 3-ml venous samples of $\mathrm{K}_{2}$-ethylendiaminotetraacetic acid (EDTA) anti-coagulated peripheral blood of four healthy, non-allergic, subjects (blood donors). Blood from four different donors was employed in each separate experiment. All subjects were informed, by physicians of the Hospital Transfusion Service, about the use of their blood samples for research purposes; they completed and signed a specific consenting form for taking the samples and data processing. Venous blood samples were processed within 2-3 h of being drawn, using a differential centrifugation step procedure. The blood was diluted to $1: 4$ in refrigerated $\left(+2^{\circ} \mathrm{C} /+8^{\circ} \mathrm{C}\right)$
HEPES modified buffer ([4-(2-hydroxyethylpiperazine-1ethanesulonic acid] $20 \mathrm{mmol} / \mathrm{l} ; \mathrm{NaCl} 127 \mathrm{mmol} / \mathrm{l} ; \mathrm{KCl}$ $5 \mathrm{mmol} / \mathrm{l}$; sodium-heparin $5 \mathrm{UI} / \mathrm{ml}, \mathrm{pH}$ 7.4) using 14-ml polypropylene round-bottom tubes, and pelleted at $700 \mathrm{~g}$ for $15 \mathrm{~min}$ in a $\left(+4^{\circ} \mathrm{C}\right)$ centrifuge. Leukocyte-rich buffycoat layers were individually collected, suspended in the cold HEPES-heparin (HBE) buffered solution and centrifuged at $400 \mathrm{~g}$ for $10 \mathrm{~min}$. Pelleted buffy-coats were washed out from supernatants, collected in a single tube, suspended in the cold HBE medium and centrifuged at $400 \mathrm{~g}$ for a further $10 \mathrm{~min}$. Finally, the cells were suspended in the refrigerated HBE buffer at $1: 4 \mathrm{v} / \mathrm{v}$ relative to starting whole blood volume. An aliquot of about $1 \mathrm{ml}$ of the above HBE-suspended cell culture was transferred to a Bayer ADVIA 2120 automated hematocytometer [31] for basophil counting and yield evaluation. Pooled buffy coats having a mean count of $8.47 \pm 2.09$ SD WBCx $103 / \mu 1$ and an estimated basophil concentration of $92.25 \pm 18.43 \mathrm{SD}$ cells $/ \mu \mathrm{l}$, were obtained. This yield corresponded to an enrichment of approximately 2.4 times relative to the starting whole blood [26]. Cells were treated with apyrogenic solutions and using sterile disposable plastic ware, and kept on ice to prevent any spontaneous activation [30]. Just before use, the cell suspension was pre-warmed for $10 \mathrm{~min}$ at $37^{\circ} \mathrm{C}$ and gently mixed.

\section{Cell treatment and activation}

A volume of $50 \mu \mathrm{l}$ of the histamine or water dilutions was added to $5-\mathrm{ml}$ round bottomed polypropylene vials containing $50 \mu \mathrm{l}$ of $2 \times$ concentrated HBE buffer (test tubes series "A") and warmed at $37^{\circ} \mathrm{C}$ for $10 \mathrm{~min}$. Then $100 \mu \mathrm{l}$ of the cell suspensions was added to each vial and incubated for $10 \mathrm{~min}$ at $37^{\circ} \mathrm{C}$. A parallel series of $5 \mathrm{ml}$-round bottomed polystyrene vials were prepared, each containing $50 \mu \mathrm{l}$ of an HBE solution supplemented with $5 \mathrm{mM} \mathrm{CaCl}_{2}$, $1 \mathrm{mM} \mathrm{MgCl}{ }_{2}$ and with the anti-IgE agonist, where indicated (final concentration $1 \mu \mathrm{g} / \mathrm{ml}$ ) (test tubes series "B"). After the 10-min incubation with histamine dilutions, $50 \mu \mathrm{l}$ of the cell suspensions from test tube series " $\mathrm{A}$ " was added into those of series " $\mathrm{B}$ ", previously brought to $37^{\circ} \mathrm{C}$. Incubation with and without anti-IgE was carried out at $37^{\circ} \mathrm{C}$ for $30 \mathrm{~min}$; the tubes were gently stirred every $10 \mathrm{~min}$ to allow proper mixing. The incubation was halted by adding $100 \mu \mathrm{l}$ of ice-cold HBE buffer supplemented with $2.8 \mathrm{mmol} / 1 \mathrm{Na}_{3}$-EDTA, and the samples put on ice until staining with monoclonal antibodies. We only included experiments that met the following criteria: (a) significant cell activation following anti-IgE treatment, expressed as a statistically significant increase of 203c MFI in triplicate samples of anti-IgE treated cells as compared with triplicate samples of untreated, resting cells and, (b) inhibition induced by histamine $2 \mathrm{C}>50 \%$ as compared 
with cell samples activated in the absence of histamine [17]. Applying these criteria, five of the seven experiments performed with histamine dilutions and five of the six experiments performed with water dilutions were selected as valid.

Staining with monoclonal antibodies and sample analysis

Staining was performed at $4^{\circ} \mathrm{C}$ for $20 \mathrm{~min}$ with $10 \mu \mathrm{l} / 200 \mu \mathrm{l}$ cell suspensions of the marker antibodies Anti-CD203c-PE, anti-CD123-PECy5, anti-CD63-FITC and with $5 \mu \mathrm{l} / 200 \mu \mathrm{l}$ cell suspensions of the marker antibodies CD45-APCCy7 and HLA-DR-PECy7. The negative controls consisted of isotype matched, directly conjugated non-specific antibodies. Soon after staining, samples underwent erythrocyte lysis with $3 \mathrm{ml}$ of a $+4^{\circ} \mathrm{C}$ refrigerated ammonium-chloride solution $\left(\mathrm{NH}_{4} \mathrm{Cl}\right.$ $155 \mathrm{mmol} / \mathrm{l} ; \mathrm{NaHCO}_{3} 10 \mathrm{mmol} / \mathrm{l}, \mathrm{Na}_{3}$ EDTA $0.10 \mathrm{mmol} /$ $1, \mathrm{pH}=7.2$ ) for $2 \mathrm{~min}$ on ice, after which the cells were pelleted at $500 \mathrm{~g}$ for $5 \mathrm{~min}$ in a refrigerated centrifuge. Supernatants were removed and the pellets gently assumption of normality, it was decided to use non-parametric tests. Since ANOVA was unsuitable due to nonnormality, for the global analysis of all the treatments, we adopted the Friedman non-parametric test for multiplerelated samples. For the comparison between control and treatment groups, we applied the two-tailed Wilcoxon nonparametric test for paired samples. The data were first of all paired by experiment: since we had three different results within each experiment, we compared the lower, the middle and the upper value of the control group with the corresponding value of the treated group. We then took all the 15 values from the five experiments on each treatment group and compared them with all the 15 values from the control (no histamine) group. Since the CD203c is constitutively expressed at low level in resting, unstimulated basophils, the net activation response to anti-IgE (dMFI) was calculated by subtracting the mean resting fluorescence expressed by unstimulated cells in each experiment from the fluorescence values of anti-IgE activated samples. Then, the percentage inhibitory effect of each dilution on the cell activation to the anti-IgE was calculated, for each experiment, according to the formula:

Mean dMFI of control (no histamine) - dMFI of histamine - treated sample

Mean dMFI of control (no histamine)

suspended in $0.5 \mathrm{ml}$ of a BD-Isoflow phoshpate saline (PBS, $\mathrm{pH}=7.4$ ) balanced buffer. Flow analysis was performed using a 488-633 nm two-laser flow cytometer (BD FACScanto). A region of low side-scatter cells was gated in the $\mathrm{CD} 45^{\mathrm{dim}}$ lymphocyte area; in this region about 500 (450-550) cellular events from each sample with a HLADR ${ }^{\text {non-expressing }} / \mathrm{CD} 123^{\text {bright }}$ phenotype were identified as basophils [26, 32]. The threshold between CD63 $3^{\text {non-expressing }}$ and CD63 $3^{\text {expressing }}$ basophils was arbitrarily set in each experiment close to the right-hand limit of the fluorescence peak of a sample of unstimulated, untreated cells. The MFI was calculated by the cytometer software by averaging the total fluorescence of the marker in the basophil gate.

\section{Statistics}

The observed MFI values for each experiment were recorded, and exploratory statistics such as the average and standard deviation were computed. Normality was checked by the Shapiro-Wilk test. The symmetry of the data was also tested, showing our experimental MFI values to be strongly right-skewed. In light of the non-symmetrical distribution of the data, which clearly did not satisfy the
The level of significance was set at $P<0.05$.

\section{Results}

Figure 1 shows a typical dot plot of basophil immunologic phenotyping and electronic capture. The HLADR-PECy7/ CD123-PECy5 plot, constructed using the CD45-dim cells in the lymphocyte area (see Sect. "Materials and methods"), made it possible to define a clearly delimited area of CD123 ${ }^{\text {bright }}$ cells not expressing HLADR, which can be identified as basophils (Fig. 1a). Basophil response toward the agonist was initially examined by evaluating twoparameter dot plots of the acquired events (Fig. 1b, c). Non-activated (resting) basophils (Fig. 1b, panel C) typically showed a very low CD63 fluorescence (MFI from 0 to $10^{3}$ units) and a low level of CD203c fluorescence (MFI from $3 \times 10^{2}$ to $3 \times 10^{3}$ units). Following $30 \mathrm{~min}$ of incubation with $1 \mu \mathrm{g} / \mathrm{ml}$ anti-IgE, only a small fraction of cells (from 3 to $15 \%$ of total basophils in the entire series of experiments) showed a CD63 bright phenotype (Fig. 1c, panels $A$ and $B$ ), while between 60 and $80 \%$ of basophils up-regulated CD203c membrane expression (Fig. 1c, panels $\mathrm{C}$ and D). From these two-parameter dot plots, a large 
Fig. 1 Human basophil gating with the BD-FACScanto flow cytometer. In a leukocytes are immunologically gated in the CD45 $5^{\text {dim }}$-lymphocyte area and electronically captured as cellular events in HLADR ${ }^{\text {non- }}$ expressing/CD123 bright twoparameter plot to distinguish them from monocytes $(Q 1)$, plasmacytoid dendritic cells (Q2) and lymphocytes (Q3). Dot plots $\mathbf{b}$ and $\mathbf{c}$ show CD63/ CD203c expression in resting and anti-IgE-activated human basophils, respectively. The cloud of resting CD203c $\mathrm{c}^{\text {low }}$ expressing /CD63 $3^{\text {negative }}$ cells (area $C$ in b) shifts to the right upon activation (c), but only a small percentage of cells express CD63 (area B in c)
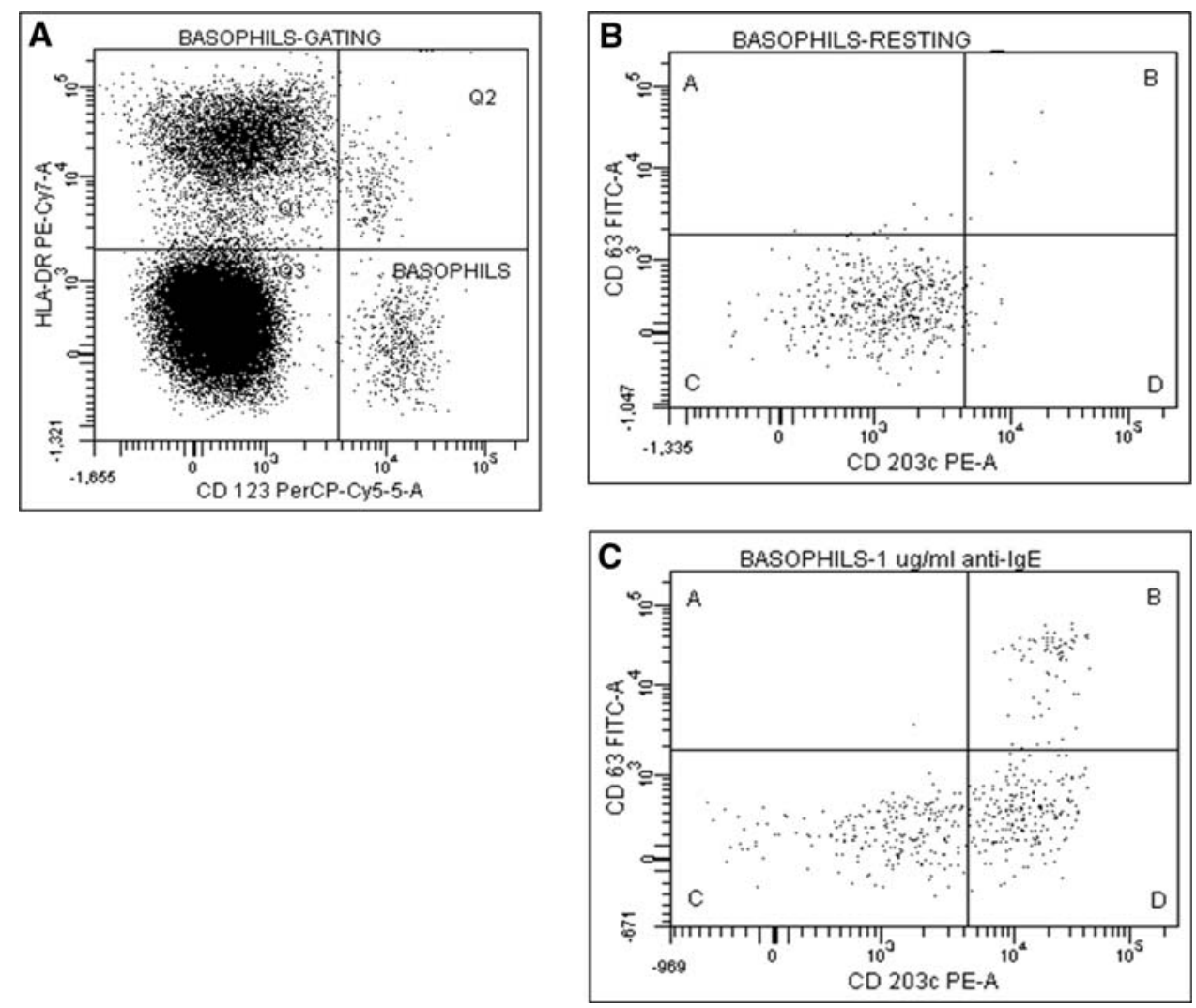

population of activated basophils expressing a CD203c $\mathrm{c}^{\text {bright }}$ phenotype was clearly evident, while most of these activated cells were negative at the CD63 staining. This confirms that CD203c is a more suitable type of marker for this type of study $[17,28]$, particularly when the extent of activation is low [26].

Table 1 reports the data of five experiments, each one done in triplicate, where basophil samples were activated in the absence and in the presence of histamine at increasing dilutions. Activation of basophils with anti-IgE caused a considerable increase in the MFI of CD203c compared with unstimulated cells (from a mean of 1812.4 units to a mean of 4389.0 units in the series of five experiments). The complete series of 15 replicates was analyzed using non-parametric tests since the distribution of values was not normal. Histamine 2C (positive control) reduced the response to 2781.7 MFI units $(P=0.001)$. A global analysis using the Friedman test for multiple samples indicated that the differences between control samples (no histamine) and the series of samples treated with the high dilutions of histamine were statistically significant $(P=0.012)$, even excluding the $2 \mathrm{C}$ test solution which was the obvious positive control in this series. The two-sample comparison with control revealed significant inhibitions of CD203c up-regulation following incubation with histamine 12C $(P=0.047), 14 \mathrm{C}(P=0.003), 15 \mathrm{C}$ $(P=0.036)$, 16C $(P=0.009)$; with histamine $13 \mathrm{C}$ the difference was not significant but very close to the limit value $(P=0.061)$.

Table 1 shows the results of five separate experiments in which succussed high dilutions of histamine were replaced with succussed high dilutions of water. For technical reasons due to the high number of tested dilutions and of replicates and in order to maintain high analytical accuracy, we could not test all histamine dilutions and all water dilutions in the same cells, so the two series of experiments reported in Table 1 were done in separate sequences, but using exactly the same protocol and the same water batch. The response of control, uninhibited samples to anti-IgE shifted from a mean of 1552.2 units to a mean of 5694.3 units and histamine $2 \mathrm{C}$ dilution (positive control for inhibition) reduced $\mathrm{CD} 203 \mathrm{c}$ expression to 3252.9 MFI units $(P=0.001)$, showing that the cells were fully sensitive to histamine, as well as the first series reported in Table 1. However, none of the water succussed dilutions showed any significant effect on the test system in two-sample comparisons with uninhibited, anti-IgE-activated sample. The Friedman test for the control and high dilutions, with the histamine $2 \mathrm{C}$ sample (positive control) omitted from the analysis, was negative $(P=0.590)$.

Table 2 shows the percentage of CD63 $3^{\text {expressing }}$ cells. These data are from the same cell samples tested for CD203c MFI and reported in Table 1. The percentage of spontaneous activation (no anti-IgE) was very low 


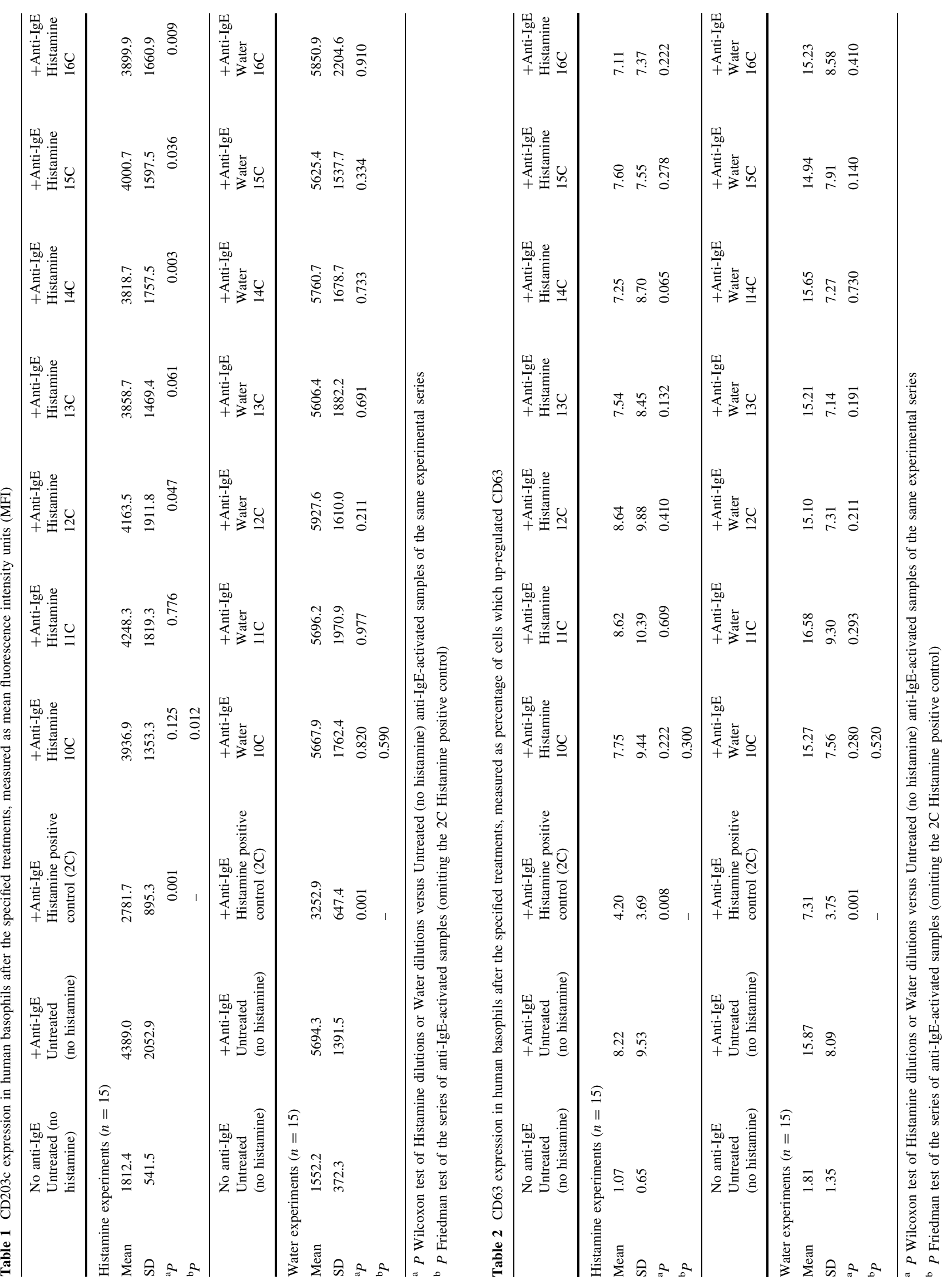


(1.07-1.81\% in histamine and water experiments, respectively), indicating that the isolation procedure yielded a basophil preparation at resting state and close to physiological conditions. Following challenge with anti-IgE, the CD63 $3^{\text {expressing }}$ cells increased to $8.22 \%$ in the series of experiments testing high histamine dilutions (2a), and to $15.87 \%$ in the series of experiments testing high water dilutions (2b). This difference between these two series of experiments, in the basophil response to anti-IgE could be explained by the very low expression of CD63 in our experimental conditions (see also Fig. 1), which is associated with high variability of data. A further factor of variability could be related to high inter-individual differences of basophil responsiveness, possibly due to seasonal variations or previous contacts with sensitizing substances, previously noted by others $[27,33]$, which may be reduced but not completely eliminated by pooling buffy-coats from different donors. Anymore, also with this marker all the cells tested were strongly inhibited by histamine at low dilution (2C), which decreased the CD63 $3^{\text {expressing }}$ cells to $4.20 \%$ (Table 2) and to $7.31 \%$ (Table 2) $(P=0.008$ and 0.001 , respectively). A few high histamine dilutions also decreased the percentage of $\mathrm{CD} 63^{\text {expressing }}$ cells (e.g. 13C, $14 \mathrm{C}, 15 \mathrm{C}, 16 \mathrm{C}$ ), but because of high variation of values this effect was not statistically significant (borderline for histamine 14C). No effects were determined by highly diluted water and succussed solutions.

Figure 2 reports the percentage inhibition by histamine and control water across the range of high dilutions tested on CD203c expression (MFI). Preliminary experiments showed that histamine (low and high dilutions) did not affect the resting MFI but only the activation response. So the percentage effect was calculated not on the absolute values, but only on the net MFI after subtracting the resting MFI. Histamine 2C (positive control) inhibition was 60.91\% $(P<0.01)$ and $59.43 \%(P<0.01)$ in the experiments testing histamine and water dilutions, respectively. There was no significant difference between the inhibition by histamine $2 \mathrm{C}$ in the two series. The dilution-effect histogram of histamine (A) shows a non-linear behaviour, with peaks at $14 \mathrm{C}$ and $16 \mathrm{C}$ dilution and paradoxically less activity at lower dilutions. No significant effects of water control dilutions (B) were observed.

\section{Discussion}

Human basophils are peripheral granulocytes involved in immune and inflammatory processes such as hypersensitivity and allergic reactions. The discovery of several basophil activation markers, such as the tetraspanin CD63 (lysosome-associated membrane protein or LAMP-3) and the ectoenzyme CD203c (or ecto-nucleotide pyrophosphatase/
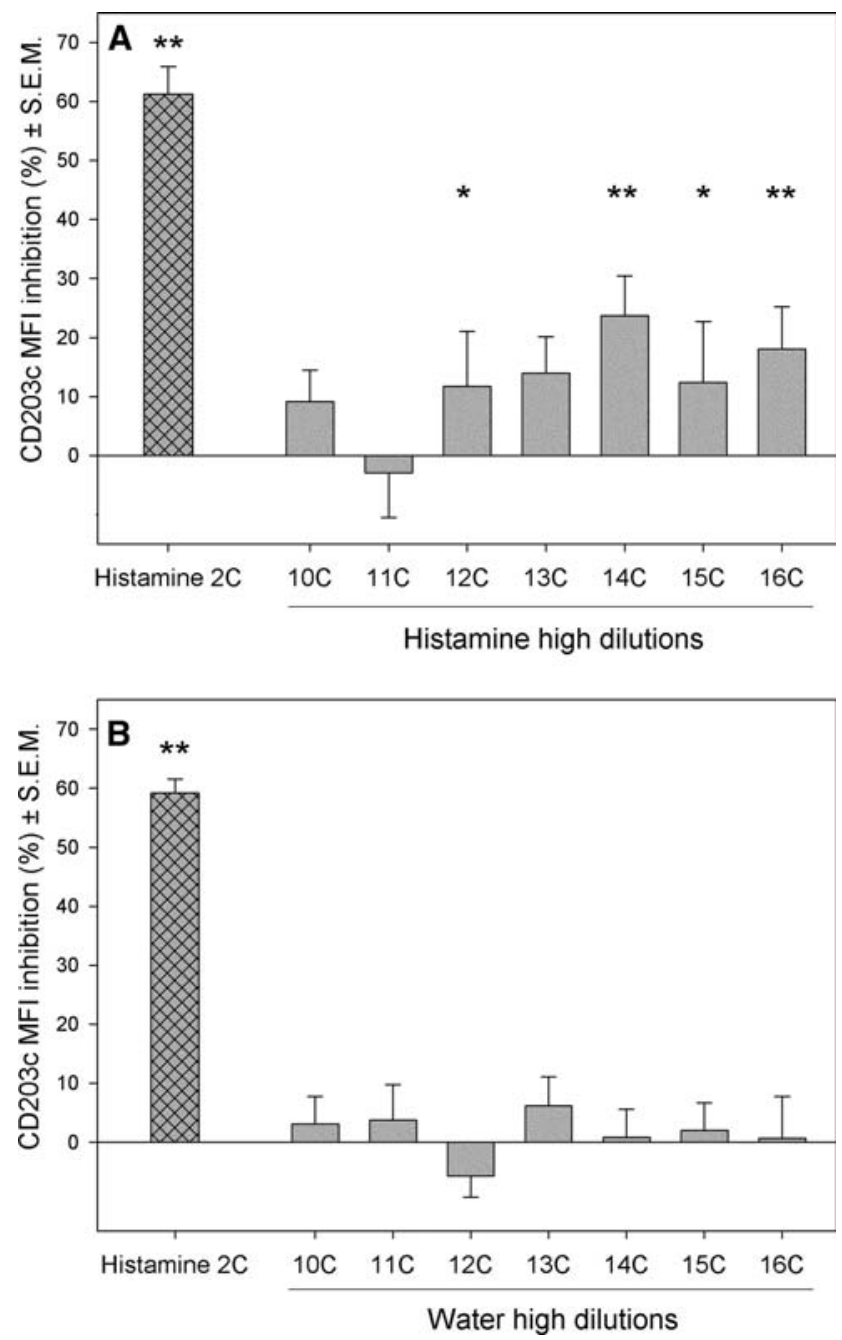

Fig. 2 Percentage inhibition of anti-IgE-activated CD203c expression in human basophils treated with high histamine dilutions (a) or with high water dilutions (b). Mean \pm SEM percentages from five experiments in (a) and from five experiments in (b) were calculated after subtracting the constitutive fluorescence of resting cells from the fluorescence of anti-IgE-activated cells, as described in "Materials and methods". $* P<0.05, * * P<0.01$ (two-tailed Wilcoxon test for paired data, comparing histamine or water dilutions with the mean of untreated controls in each experiment). CD203c MFI values of the uninhibited activation were $2576.6 \pm 543.3$ SEM in the histamine dilution experiments (a) and $4142.1 \pm 348.4$ SEM in the water dilution experiments (b)

phosphodiesterase or E-NPP3), has made it possible to study cellular function by observing the up-regulation or downregulation of membrane molecules in response to various physiological agonists, antagonists or other soluble regulators $[34,35]$, using a standardized cytometric approach.

Among the various soluble factors able to regulate basophil function, a considerable body of literature has been devoted to histamine, a well-known mediator of inflammation produced by basophils and mast cells. Histamine at high doses acts as a downstream regulator by 
interacting with $\mathrm{H} 2$-receptors, thus inhibiting basophil release of mediators and degranulation [36]; it is therefore very intriguing that the same effect was observed with highly diluted histamine but not with highly diluted histidine [11], and that it could be inhibited by the H2antagonist cimetidine [37]. Multicentre analyses have partially confirmed these findings [10], but other groups also report some negative results [19].

In this work we were able to reproduce evidence for the effects of high histamine dilutions on anti-IgE-triggered basophil activation, specifically evaluated by the CD203 MFI, using a standardized approach with the following characteristics: (a) the method of cell handling prevents spontaneous basophil activation and interference between the anti-IgE-FITC tracer and the agonist used [14]; (b) the problems of subjective evaluation are avoided through use of flow cytometry and a system of multiple intra-series replication; (c) use of highly pure compounds and parallel comparisons with control pure water dilutions; d) adoption, for the first time in this field, of a mechanical shaking method (succussion in homeopathic terms) with standardized duration and frequency of strokes.

The resultant dose-effect curve displayed a very unusual behaviour, whose underlying mechanism remains to be investigated. It is worth noting that the range of active dilutions is very close to the $16 \mathrm{C}$ dilution reported elsewhere [11, 15], although Brown and Ennis [13] instead reported inhibitory activities for $10 \mathrm{C}$ and $13 \mathrm{C}$, but not for the $12 \mathrm{C}, 14 \mathrm{C}$ and $16 \mathrm{C}$ dilutions.

The lack of significant inhibition of CD63 expression by histamine at high dilutions in our experimental conditions (Table 2) may apparently be in disagreement with the data of $\mathrm{CD} 203 \mathrm{c}$ and with the findings of other authors who detected high-dilution effects also using CD63 marker [11]. However, this discrepancy may be interpreted as due to the lower sensitivity of this parameter to inhibition by high dilutions or otherwise as a result of the high variability of this response, because of the low percentage of cells expressing CD63 in our test system. In this study we chose to use low doses of agonist, a condition in which cells were consistently activated, as noted by the increase of CD203c MFI, but very low percentage $(8-15 \%)$ of cells expressed CD63, which is less responsive to low doses of anti-IgE (Fig. 1a). As a consequence, the high-dilution effects were much more clearly and consistently observable by evaluating the CD203c marker. We have previously shown that the chemotactic peptide fMLP, at very low dose, causes upregulation of $\mathrm{CD} 203 \mathrm{c}$ but not of CD63 [26], and this observation is consistent with the finding that $\mathrm{CD} 203 \mathrm{c}$ reflects the priming events triggered by interleukin-3, which occur previously to degranulation events [28]. Moreover, CD203c expression in not inhibited by phosphatidylinositol-3-kinase inhibitors, as is CD63 [29]. Thus, small changes in the experimental conditions used or subtle distinction of the signal transduction mechanisms of the two markers expression could differentiate the results of high dilution experiments obtained by different authors in this model.

Although a variety of gating protocols and different experimental conditions-in terms of the type of dilution/ succussion or shaking procedures and solvent used-have been explored [13, 38], most of the studies done so far did not include succussed water controls. Research on extremely sensitive systems and very high dilutions of substances suggests that trace elements, as well as container materials, storage durations and shaking methods, may influence the results [39]. Therefore, suitable water controls prepared in an identical manner and subjected to the same storage time should be used. These considerations, coupled with the highly controversial nature of the phenomenon, which would have significant pharmacological implications but is often judged to be improbable and implausible from a conventional scientific perspective [24, 40, 41], make it all the more important that more replications should be done independently to establish models that are stable across laboratories and teams [22].

The possible biological mechanism(s) underlying the regulatory processes affected by high histamine dilutions remain to be elucidated. These findings suggest that the high dilutions of histamine, which are better observed through CD203c expression, might affect some subtle and early level of signal transduction, similar to the priming effects of very low doses, instead of causing a general inhibition of cell responses. Using a surface plasmon resonance biosensor, it has recently been shown that basophil activation by anti-IgE, even in the absence of exocytosis, is associated with changes in the refractive index of the evanescent field [42]; such changes reflect any changes in molecular mass distribution in the field a few $100 \mathrm{~nm}$ from the surface of the cells. It is thus tempting to speculate that changes in the water structure produced by the dilution/ succussion procedure in the presence of histamine might influence this crucial layer of water surrounding the cell and hence the processes of signal detection and transduction. Further studies are needed to confirm whether this hypothesis is applicable to the high-dilution effects observed on human basophils.

Our results confirm and build upon the hypothesis that high dilutions of biologically active compounds may indeed have an effect which mimics that of lower dilutions/higher doses. So far there is no satisfactory or uniting theoretical explanation for these observations, but recent evidence seems to point to organization of the solvent water on a mesoscopic scale: the nano-heterogenous structure of water can be determined by interactive phenomena such as coherence [43, 44], epitaxy [45], temperature-pressure 
processes during strong agitation, and formation of colloidal nanobubbles containing gaseous inclusions of oxygen, nitrogen, carbon dioxide, silica, and possibly the remedy source material [46-48]. So far, nuclear magnetic resonance (NMR) studies of homeopathic solutions have yielded controversial data, but recently high histamine dilutions (beyond $10^{-20} \mathrm{~mol} / \mathrm{l}$ ) were distinguished from water solvents on the basis of their NMR relaxation rates [49]; moreover, after heating of the histamine samples, all the relaxation observed as a function of dilution vanished. It was suggested that histamine molecules might act as nucleation centres, amplifying the formation of stable supramolecular structures, involving nanobubbles of atmospheric gases and highly ordered water around them. In the future, the possible existence and the nature of clathratelike hydrate nanostructures formed during the dilution and succussion process might be explained by cluster science, in which different geometrical structures of clusters composed of the same chemical species may differ in their chemical reactivity [50]. These unusual properties of high dilutions, which merit further investigation, are potentially relevant not just to homeopathic pharmaceutical practice, but also to basic research into cell sensitivity to regulation.

Acknowledgements The study was financed by a grant from Laboratories Boiron srl, Milano (Italy), and in part by the Italian Ministry of Education, University and Research (60\% funds). The authors wish to thank Dr Luigi Marrari for his advice and cooperation.

Open Access This article is distributed under the terms of the Creative Commons Attribution Noncommercial License which permits any noncommercial use, distribution, and reproduction in any medium, provided the original author(s) and source are credited.

\section{References}

1. Bellavite P, Conforti A, Pontarollo F, Ortolani R. Immunology and homeopathy. 2. Cells of the immune system and inflammation. Evid Based Complement Alternat Med. 2006;3(1):13-24.

2. Bellavite P, Conforti A, Ortolani R. Immunology and homeopathy. 3. Experimental studies on animal models. Evid Based Complement Alternat Med. 2006;3:171-86.

3. Davenas E, Beauvais F, Amara J, Robinson M, Miadonna A, Tedeschi A, et al. Human basophil degranulation triggered by very dilute antiserum against IgE. Nature. 1988;333:816-8.

4. Maddox J, Randi J, Stewart WW. "High-dilution" experiments a delusion. Nature. 1988;334:287-90.

5. Ovelgonne JH, Bol AWJM, Hop WCJ, Van Wijk R. Mechanical agitation of very dilute antiserum against $\operatorname{IgE}$ has no effect on basophil staining properties. Experientia. 1992;48:504-8.

6. Hirst SJ, Hayes NA, Burridge J, Pearce FL, Foreman JC. Human basophil degranulation is not triggered by very dilute antiserum against human IgE. Nature. 1993;366:525-7.

7. Cherruault Y, Guillez A, Sainte-Laudy J, Belon P. E'tude mathematique et statistique des effets de dilutions successives de chlorhydrate d'histamine sur la réactivité des basophiles humains. Bioscience. 1989;7:63-72.
8. Sainte-Laudy J, Sambucy JL, Belon P. Biological activity of ultra low doses I. Effect of ultra low doses of histamine on human basophil degranulation triggered by $\mathrm{D}$. pteronissinus extract. In: Doutremepuich C, editor. ultra low doses. London: Taylor and Francis; 1991. p. 127-38.

9. Sainte-Laudy J, Belon P. Inhibition of human basophil activation by high dilutions of histamine. Agent Actions (Inflamm Res). 1993;38:C245-7.

10. Belon P, Cumps J, Ennis M, Mannaioni PF, Sainte-Laudy J, Roberfroid M, et al. Inhibition of human basophil degranulation by successive histamine dilutions: results of a European multicentre trial. Inflamm Res. 1999;48:S17-8.

11. Belon P, Cumps J, Ennis M, Mannaioni PF, Roberfroid M, Sainte-Laudy J, et al. Histamine dilutions modulate basophil activation. Inflamm Res. 2004;53:181-8.

12. Sainte-Laudy J, Belon P. Analysis of immunosuppressive activity of serial dilutions of histamine on human basophil activation by flow cytometry. Inflamm Res. 1996;45:S33-4.

13. Brown V, Ennis M. Flow-cytometric analysis of basophil activation: inhibition by histamine at conventional and homeopathic concentrations. Inflamm Res. 2001;50:S47-8.

14. Lorenz I, Schneider EM, Stolz P, Brack A, Strube J. Sensitive flow cytometric method to test basophil activation influenced by homeopathic histamine dilutions. Forsch Komplementarmed Klass Naturheilkd. 2003;10(6):316-24.

15. Sainte-Laudy J, Belon P. Use of four different flow cytometric protocols for the analysis of human basophil activation. Application to the study of the biological activity of high dilutions. Inflamm Res. 2006;55(Suppl 1):S1-2.

16. Sainte-Laudy J, Boujedaini N, Belon P. Confirmation of biological effects of high dilutions. Effects of submolecular concentrations of histamine and 1-, 3- and 4-methylhistamines on human basophil activation. Inflamm Res. 2008;57(Suppl 1): S27-8.

17. Sainte-Laudy J, Belon P. Improvement of flow cytometric analysis of basophil activation inhibition by high histamine dilutions. A novel basophil specific marker: CD 203c. Homeopathy. 2006;95(1):3-8.

18. Sainte-Laudy J. Stimulatory effect of high dilutions of histamine on activation of human basophils induced by anti-IgE. Inflamm Res. 2001;50(Suppl 2):S63-4.

19. Guggisberg AG, Baumgartner SM, Tschopp CM, Heusser P. Replication study concerning the effects of homeopathic dilutions of histamine on human basophil degranulation in vitro. Complement Ther Med. 2005;13(2):91-100.

20. Linde K, Jonas WB, Melchart D, Worku F, Wagner H, Eitel F. Critical review and meta-analysis of serial agitated dilutions in experimental toxicology. Hum Exp Toxicol. 1994;13:481-92.

21. Jonas WB, Kaptchuk TJ, Linde K. A critical overview of homeopathy. Ann Intern Med. 2003;138:393-9.

22. Witt CM, Bluth M, Albrecht H, Weisshuhn TE, Baumgartner S, Willich SN. The in vitro evidence for an effect of high homeopathic potencies-a systematic review of the literature. Complement Ther Med. 2007;15(2):128-38.

23. Van Wijk R. The in vitro evidence for an effect of high homeopathic potencies - a systematic review of the literature. Complement Ther Med. 2007;15(2):139-41.

24. Novella S, Roy R, Marcus D, Bell IR, Davidovitch N, Saine A. A debate: homeopathy-quackery or a key to the future of medicine? J Altern Complement Med. 2008;14(1):9-15.

25. Falus A. Homeopathy and high dilutions-is there a real effect? Inflamm Res. 2004;53(5):179-80.

26. Chirumbolo S, Vella A, Ortolani R, DeGironcoli M, Solero P, Tridente G, et al. Differential response of human basophil activation markers: a multi-parameter flow cytometry approach. Clin Mol Allergy. 2008;16(6):12. 
27. Valent P, Hauswirth AW, Natter S, Sperr WR, Bühring HJ, Valenta R. Assays for measuring in vitro basophil activation induced by recombinant allergens. Methods. 2004;32(3):265-70.

28. Boumiza R, Monneret G, Forissier M, Savoye J, Gutowski MC, Powell WS, et al. Marked improvement of the basophil activation test by detecting CD203c instead of CD63. Clin Exp Allergy. 2003;33:259-65.

29. de Weck AL, Sanz ML, Gamboa PM, Aberer W, Bienvenu J, Blanca M, et al. Diagnostic tests based on human basophils: more potentials and perspectives than pitfalls. Int Arch Allergy Immunol. 2008;146(3):177-89.

30. Ocmant A, Peignois Y, Mulier S, Hanssens L, Michils A, Schandens L. Flow cytometry for basophil activation markers: the measurement of CD203c up-regulation is as reliable as CD63 expression in the diagnosis of cat allergy. J Immunol Methods. 2007;320:40-8.

31. Harris N, Jou JM, Devoto G, Lotz J, Pappas D, Wranovics D, et al. Performance evaluation of the ADVIA 2120 hematology analyzer: an international multicenter clinical trial. Lab Hematol. 2005; 11:62-70.

32. Han X, Jorgensen JL, Brahmandam A, Schlette E, Huh YO, Shi $\mathrm{Y}$, et al. Immunophenotypic study of basophils by multiparameter flow cytometry. Arch Pathol Lab Med. 2008;132:813-9.

33. Saporta M, Kamei S, Persi L, Bousquet J, Arnoux B. Basophil activation during pollen season in patients monosensitized to grass pollens. Allergy. 2001;56:442-5.

34. Knol E, Mulf F, Jansen H, Calafat J, Roos D. Monitoring human basophil activation via CD63 monoclonal antibody 435. J Allergy Clin Immunol. 1991;88:328-38.

35. Buhring J, Streble A, Valent P. The basophil-specific ectoenzyme E-NPP3 (CD203c) as a marker for cell activation and allergy diagnosis. Int Arch Allergy Immunol. 2004;133:317-29.

36. Masini E, Blandina P, Brunelleschi S, Mannaioni PF. Evidence for H2-receptor-mediated inhibition of histamine release from isolated rat mast cells. Agents Actions. 1982;12(1-2):85-8.

37. Sainte-Laudy J, Belon P. Application of flow cytometry to the analysis of the immunosuppressive effect of histamine dilutions on human basophil activation: effect of cimetidine. Inflamm Res. 1997;46:S27-8.

38. Lorenz I, Schneider EM, Stolz P, Brack A, Strube J. Influence of the diluent on the effect of highly diluted histamine on basophil activation. Homeopathy. 2003;92(1):11-8.
39. Witt CM, Ludtke R, Weisshuhn TE, Quint P, Willich SN. The role of trace elements in homeopathic preparations and the influence of container material, storage duration, and potentisation. Forsch Komplement Med. 2006;13(1):15-21.

40. Shang A, Huwiler-Müntener K, Nartey L, Jüni P, Dörig S, Sterne JAC, et al. Are the clinical effects of homoeopathy placebo effects? Comparative study of placebo-controlled trials of homoeopathy and allopathy. Lancet. 2005;366:726-32.

41. Fisher P. On the plausibility of homeopathy. Homeopathy. 2008;97(1):1-2.

42. Suzuki H, Yanase $Y$, Tsutsui $T$, Ishii $K$, Hiragun T, Hide M. Applying surface plasmon resonance to Monitor the IgE-mediated activation of human basophils. Allergol Int. 2008;57(4): 347-58.

43. Del Giudice E, Preparata G. Coherence electrodynamics in water. In: Schulte J, Endler C, editors. Fundamental research in ultrahigh dilution and homeopathy. Dordrecht: Kluwer; 1998. p. $89-100$.

44. Smith CW. Quanta and coherence effects in water and living systems. J Altern Complement Med. 2004;10(1):69-78.

45. Rao ML, Roy R, Bell IR, Hoover R. The defining role of structure (including epitaxy) in the plausibility of homeopathy. Homeopathy. 2007;96(3):175-82.

46. Demangeat JL, Gries P, Poitevin B, Droesbeke JJ, Zahaf T, Maton F, et al. Low-field NMR water proton longitudinal relaxation in ultrahighly diluted aqueous solutions of silica-lactose prepared in glass material for pharmaceutical use. Appl Magn Reson. 2004;26:465-81.

47. Elia V, Niccoli M. New physico-chemical properties of extremely diluted aqueous solutions. J Thermal Anal Calorim 2004;(75): 815-36.

48. Chaplin MF. The memory of water: an overview. Homeopathy. 2007;96(3):143-50.

49. Demangeat JL. NMR water proton relaxation in unheated and heated ultrahigh aqueous dilutions of histamine: evidence for an air-dependent supramolecular organization of water. J Mol Liquids 2008; doi:10.1016/j.molliq.2008.07.013.

50. Roy R, Bell IR, Hoover. The structure of liquid water. Novel insights from materials research; potential relevance to homeopathy. Mat Res Innovat. 2005;9(4):98-103. 